\title{
Death by super-resolution imaging
}

\begin{abstract}
Researchers report that the illumination intensities used in super-resolution imaging can irreversibly damage live cells.

Super-resolution imaging methods such as direct stochastic optical reconstruction microscopy (dSTORM), photoactivated localization microscopy (PALM) and stimulated emission depletion (STED) microscopy can provide highly detailed views of structures within cells. However, these methods require high doses of light, which can have unwanted effects, known as photodamage and phototoxicity, on biological samples. Markus Sauer at the University of Würzburg, along with two graduate students in his laboratory, Sina Wäldchen and Julian Lehmann, sought to carefully characterize such effects in live cells.
\end{abstract}

The research was motivated by conflicting reports regarding phototoxicity in the field of super-resolution fluorescence microscopy. "Some people claimed that very low light doses, even sunlight, can seriously kill cells," says Sauer, whereas "other researchers use orders-of-magnitude higher light doses in living cells and even in living mouse brain and claim that they do not observe any signs of photodamage." The team was interested in the effects of fluorophores, which are known to generate harmful reactive oxygen species, on phototoxicity. They were also interested in whether direct interaction of light with cells can cause damage, which often goes unmentioned. "We thought somebody should shed light on these obvious discrepancies and remove the uncertainty," recalls Sauer.

The team systematically studied the effects of different light treatments on cells grown in culture. In one experiment, they treated cells grown under different conditions with a range of light intensities. A day later, they examined the effects of the light exposure by quantifying the numbers of

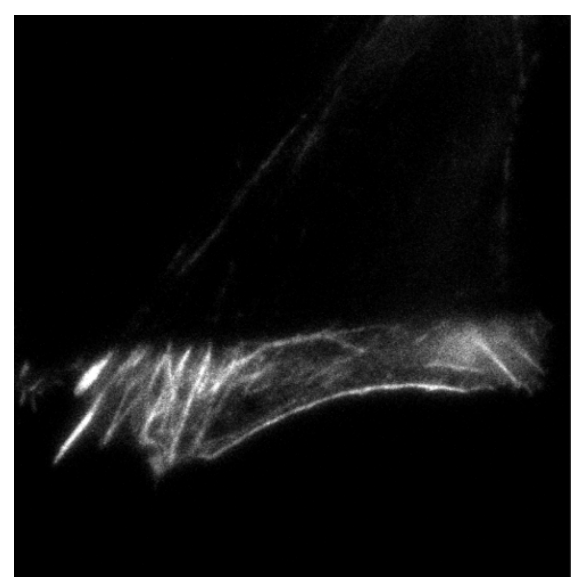

Image of a 'frozen' mammalian cell where the top portion was treated by light, destroying the actin network. Reprinted from Wäldchen et al. (2015).

dead and living cells. Some of their results were unexpected. In particular, Sauer says he was surprised that "dyes contribute to phototoxicity, but it is mainly the direct interaction of the light with other intrinsic cellular molecules which is responsible for damage." They found that transfection can increase cells' photosensitivity relative to that of untreated controls, as can the introduction of a fluorophore. They also discovered that cell lines differ in their photosensitivity, with the commonly used HeLa cell line being robust relative to the other tested lines.

The team also tested whether wavelength has a role in photodamage. They compared several wavelengths of light at a range of light intensities and found striking results. They discovered that near-UV light is extremely phototoxic, even at low intensities, whereas far-red light causes minimal phototoxicity, even at high intensities. A surprising result of this analysis was the finding that a small change in wavelength can have a large effect on phototoxicity.
Sauer notes that just the 26-nm difference between $488 \mathrm{~nm}$ and $514 \mathrm{~nm}$ can be the difference between life and death for cells being imaged.

Sauer wants a take-home message from this work to be that "users should carefully dose the use of wavelengths shorter than 500 $\mathrm{nm}$ for photoactivation or photoconversion, even at very low intensities. The longer the irradiation wavelength, the better the survival chances of cells." This may be particularly relevant to PALM experiments, which use near-UV light to photoactivate fluorophores over multiple rounds of imaging.

The authors made other important observations in the course of their experiments. For example, they found that after irradiation, some cells underwent apoptosis before death, whereas others bypassed that step. These latter, so-called frozen cells were immediately killed and fixed in place by the light treatment. Further analysis showed that, despite looking unchanged, the membrane and cytoskeleton of frozen cells were destroyed by the light treatment. These results suggest that examination for phototoxic effects should not be limited to phenotypic assessment immediately after imaging.

These results have direct implications for the design and implementation of live-cell super-resolution microscopy experiments, and they can also be used to guide the design of improved tools. Sauer believes that improved far-red dyes for super-resolution microscopy and better tools for site-specific labeling of proteins in live cells will be necessary for live-cell imaging at the nanoscale in the future.

\section{Rita Strack}

\section{RESEARCH PAPERS}

Wäldchen, S. et al. Light-induced cell damage in live-cell super-resolution microscopy. Sci. Rep. 5 , 15348 (2015). 